\title{
Fabrication and Testing of Antimony Doped Bismuth Tri-Iodide Semiconductor Gamma-Ray Detectors
}

\author{
Sasmit S. Gokhale ${ }^{\mathrm{a}, 1}$, HyukSu Han ${ }^{\mathrm{b}}$, Oswaldo Pelaez ${ }^{\mathrm{a}}$, James E. Baciak ${ }^{\mathrm{a}}$, Juan C. Nino ${ }^{\mathrm{b}}$, and \\ Kelly A. Jordan ${ }^{\mathrm{a}}$ \\ ${ }^{a}$ Nuclear Engineering program at the Department of Materials Science and Engineering, \\ 100 Rhines Hall, \\ University of Florida, \\ Gainesville, FL 32611 USA \\ ${ }^{b}$ Department of Materials Science and Engineering, \\ 100 Rhines Hall, \\ University of Florida, \\ Gainesville, FL 32611 USA \\ E-mail: sasmitg@ufl.edu \\ Tel: +1-352-871-9034
}

\begin{abstract}
Antimony $(\mathrm{Sb})$ doped bismuth tri-iodide $\left(\mathrm{BiI}_{3}\right)$ radiation detectors were fabricated from large single crystals that were grown using the modified vertical Bridgman technique. Detectors were prepared by subjecting the crystal surfaces to different mechanical and chemical treatments. Surface quality of the detectors was evaluated using optical microscopy. The influence of surface quality on detector performance was analyzed by measuring the leakage current for each of the detectors. The radiation response of the detectors was measured using an Americium $\left({ }^{241} \mathrm{Am}\right)$ gamma-ray source at room temperature. The first successful use of $\mathrm{BiI}_{3}$ detectors for gamma-ray spectroscopy is reported here with energy resolution of $7.5 \%$ at $59.5 \mathrm{keV}$. The mobility-lifetime product for electrons was also estimated to be about $5.2 \times 10^{-4} \mathrm{~cm}^{2} / \mathrm{V}$.
\end{abstract}

KEYWORDS - Radiation detector, bismuth tri-iodide, surface treatment, room temperature semiconductor detector, gamma-ray detector.

${ }^{1}$ Corresponding Author 


\section{Introduction}

Bismuth tri-iodide demonstrates a number of properties that are apt for room temperature semiconductor radiation detection, and especially gamma ray spectroscopy. The high atomic number $\left(Z_{\mathrm{Bi}}=83\right.$ and $\left.\mathrm{Z}_{\mathrm{I}}=53\right)$ and the relatively high density $\left(5.78 \mathrm{~g} / \mathrm{cm}^{3}\right)$ causes the material to have good photon stopping power [1], while the large band-gap (1.67 eV [2]) allows it to function as a room temperature radiation detector without cooling.

There are several factors that affect the detector response to radiation. Some of these factors such as the material atomic number and charge carrier mobility and lifetime are inherent to the detector crystal, while extrinsic factors such as impurities and detector surface quality also affect the performance of radiation detectors [3]. Generally detector performance can be improved by controlling these factors. $\mathrm{BiI}_{3}$ has a layered crystal structure with weak van der Waals bonding between the layers, and therefore the crystals are mechanically soft [4] and precautions have to be taken to prevent damage during detector fabrication.

Detector fabrication is carried out in a number of steps, which involves cutting the crystal boule, polishing the surfaces, and subsequently etching the polished surfaces. Each of these steps is aimed at eliminating the surface damage resulting from the previous steps. The surface damage that results from cutting the detector crystal may enhance the surface leakage current by providing more conductive paths and altered electric field distribution, which results in an increase in noise. It can also enhance carrier recombination by providing more carrier trapping sites, which reduces the charge collected at the electrodes [5].

Mechanical polishing of the surfaces followed by chemical etching are two important steps in the fabrication of detectors. Mechanical polishing removes the large surface distortions arising during cutting; however it does not leave the surface defect free. Chemical etching is carried out to remove the residual damage after mechanical polishing. Other investigations have reported the effect of surface treatment on the surface quality of semiconductor detectors [5], [6], [7], and [8]. These studies have shown that the leakage current is correlated to the surface morphology. Since leakage current is a major component of detector noise, it is important to optimize the detector fabrication process to produce detectors with good response.

Recently, Han et al. have demonstrated that Sb doping can effectively improve the electrical characteristics of $\mathrm{BiI}_{3}$ single crystals [9]. It was observed that the resistivity of the doped $\mathrm{BiI}_{3}$ crystals was on the order of $10^{10} \Omega-\mathrm{cm}$. This value of resistivity is comparable to the resistivity of the $\mathrm{BiI}_{3}$ platelets grown by physical vapor deposition (PVD) [10], [11], and [12] and is orders of magnitude higher than the value of resistivity of undoped $\mathrm{BiI}_{3}$ detectors reported in our previous work [13]. Resistivity values in the range of $10^{10} \Omega$-cm were also reported for $\mathrm{BiI}_{3}$ crystals grown by the Bridgman method, as well as for crystals grown by vapor transport [14], [15], and [16]. Additionally it is theorized that the Sb doping may delay the onset of detector polarization and as a result the detectors could be operated for longer duration.

In this work $\mathrm{Sb}$ doped $\mathrm{BiI}_{3}$ detectors were subjected to the various surface treatment steps and their surface quality was evaluated. The electrical characteristics and the radiation response of the treated detectors were measured. A number of the detectors showed response to gamma-ray 
irradiation. This was the first instance where $\mathrm{BiI}_{3}$ detectors were used to record gamma-ray spectra which could be spectrally resolved. The radiation response measurement was performed at room temperature using a ${ }^{241} \mathrm{Am}$ sealed gamma-ray source.

\section{A. Crystal Growth}

\section{Experimental Techniques}

$\mathrm{Sb}$ doped $\mathrm{BiI}_{3}$ single crystals were grown by the modified vertical Bridgman method. Pyrex glass ampoule was selected as the growth chamber due to relatively low annealing $\left(=560^{\circ} \mathrm{C}\right)$ and softening point $\left(=815^{\circ} \mathrm{C}\right)$, however those temperatures are still higher than the maximum temperature $\left(=440^{\circ} \mathrm{C}\right)$ for the $\mathrm{BiI}_{3}$ single crystal growth process. Customized Pyrex glass ampoules (inner diameter $=1.905 \mathrm{~cm}$, tip length $\cong 5 \mathrm{~cm}$, and tip angle $\cong 70^{\circ}$ ) were purchased from Southern Scientific, Inc. The Pyrex ampoule was cleaned with glassware cleaning solution (Decon Contrad ${ }^{\circledR} 70$ ) to remove residual organics. The ampoule was soaked in the solution overnight under a fume food, and it was then rinsed with DI water $(\rho \cong 17.0 \mathrm{M} \Omega-\mathrm{cm})$ followed by baking in a drying oven $\left(120^{\circ} \mathrm{C}\right)$ overnight.

Typical loads of $20 \mathrm{~g}$ of $\mathrm{BiI}_{3}$ powder (99.999 \%, MV laboratory Inc.) with appropriate amount of $\mathrm{SbI}_{3}(99.9 \%$, Strem Chemicals Inc.) based on the doping concentration were weighed and loaded in the ampoule. $\mathrm{SbI}_{3}$ was added to the ampoule first followed by the $\mathrm{BiI}_{3}$ powder. The ampoule was then evacuated to a pressure of 0.03 torr by using mechanical roughing pump, and then molecular turbo pump to finally evacuate the ampoule to $7.5 \times 10^{-5}$ torr. The vacuumed ampoule was then sealed by using a propane hand torch. The sealed ampoule was vertically mounted on a standing steel frame using galvanized steel wires, and placed in programmable 24 multi heating zone furnaces (EDG-13, Mellen Company). The previously optimized growth condition, such as temperature gradient of $10^{\circ} \mathrm{C} / \mathrm{cm}$ and growth rate of $0.5 \mathrm{~mm} / \mathrm{h}$, were utilized for the single crystal growth process [13].

In order to determine optimal doping concentration, a series of $\mathrm{BiI}_{3}$ single crystals with 0.5 at $\%, 1.0$ at $\%$, and 5.0 at $\% \mathrm{Sb}$ were grown. Detectors were then prepared from each crystal and electrical properties, such as bulk resistivity and leakage current were measured and compared. The gold electrodes sputtered onto the detectors exhibited ohmic behavior. As can be seen from Figure 1(a), the resistivity of the detectors doped with 0.5 at $\%$ and 1.0 at $\% \mathrm{Sb}$ was of the order of $10^{10} \Omega$-cm $\left(3.48 \times 10^{10} \Omega\right.$-cm for 0.5 at $\%$ and $1.7 \times 10^{10} \Omega$-cm for 1 at $\left.\%\right)$, while the 5 at $\%$ doped material had lower resistivity at around $3.75 \times 10^{9} \Omega$-cm.

In addition, Figure 1(b) shows the comparison of the leakage current density in the various $\mathrm{Sb}$ doped $\mathrm{BiI}_{3}$ single crystal detectors. The detectors were biased such that the electric field was held constant at $100 \mathrm{~V} / \mathrm{cm}$ and the leakage current was measured over a period of time. It can be seen that the leakage current density appears to change over time. This change may be attributed to polarization effects. Polarization may be caused due to a change in the internal electric field over time due to defects, traps, or impurities. Similar polarization phenomenon has been observed in other detector materials such as $\mathrm{HgI}_{2}$ and $\mathrm{TlBr}$ [17] - [18]. The effect of polarization on the radiation response of the detectors is discussed later on in the manuscript. The lowest leakage 
current was observed in the 0.5 at $\%$ doped $\mathrm{BiI}_{3}$ detector. A detector with the higher resistivity and low leakage current should have a better response to radiation since a lower dark current and a higher signal to noise ratio can be achieved. From Figure 1(b) it can

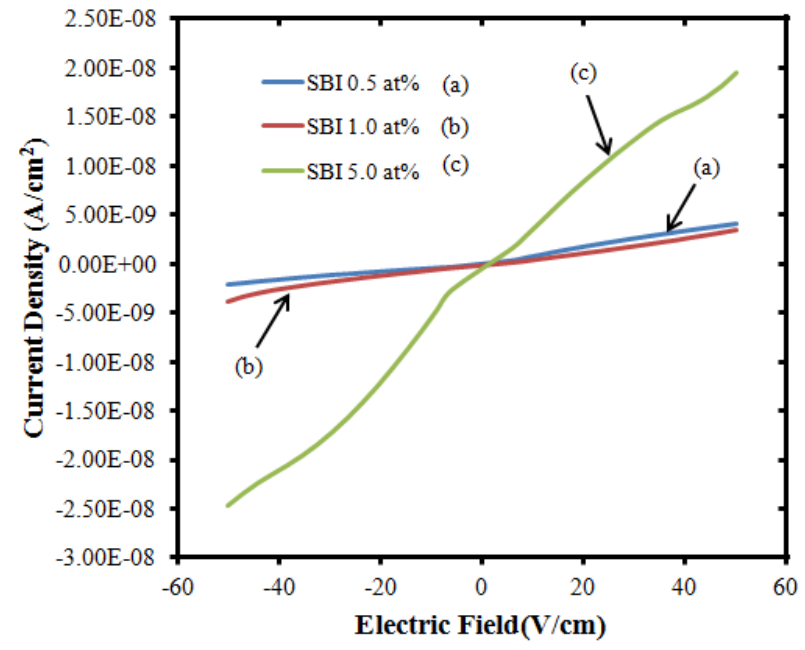

(a)

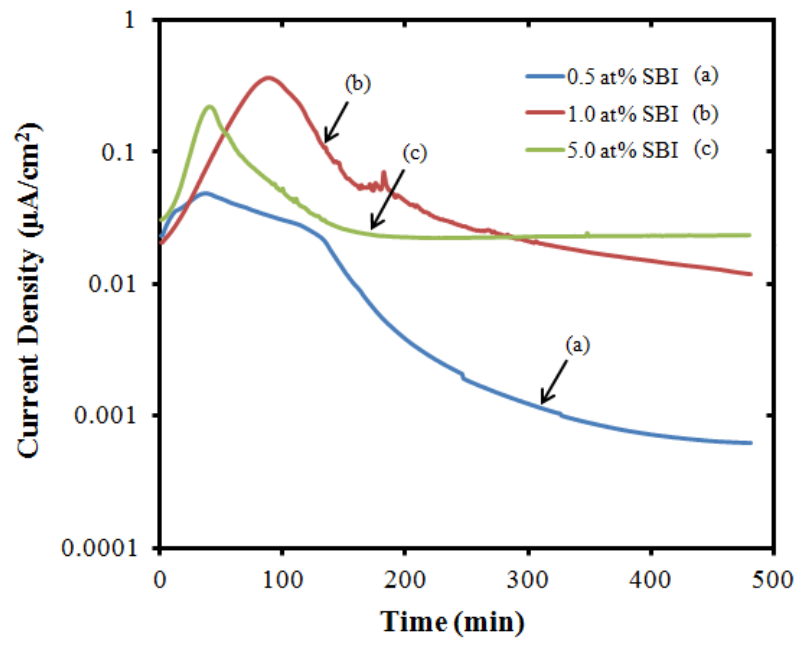

(b)

Figure 1. (a) IV characterization and (b) leakage current vs. time measurements for $\mathrm{Sb}$ doped $(0.5,1.0$, and 5.0 at $\%) \mathrm{BiI}_{3}(\mathrm{SBI})$ single crystals.

The measurements were repeated on more than eight detectors harvested from the different crystal boules to confirm the reproducibility of the results. The trends seen in Figures 1a and $1 \mathrm{~b}$ were consistently observed in all the detectors fabricated from the different doped crystals i.e. the 0.5 at $\% \mathrm{Sb}$ doped detectors had higher resistivity and lower leakage current that the detectors with other doping concentrations. Therefore, $\mathrm{Sb}(0.5$ at $\%)$ doped $\mathrm{BiI}_{3}$ was selected as it was the most promising compound for growing single crystals and fabricating radiation detectors, and the following results and discussion are only focused on this compound.

\section{B. Detector Fabrication}

Detectors were fabricated by cutting the as grown crystal into parallelepipeds using a diamond wire saw. Any mechanical stress caused due to cutting and handling the crystals can increase the concentration of intrinsic structural defects by local deformation. This limits the performance of the detectors [19]. Therefore the crystals were cut slowly, using mineral oil for lubrication to minimize damage. The wire saw cuts were made in a direction perpendicular to the [001] plane. The [001] plane is the cleavage plane, and since $\mathrm{BiI}_{3}$ has a layered structure with weak van der Waals bonding between the layers, the crystals cleave easily along the cleavage plane.

After cutting, the crystals were then polished with abrasive slurries. Polishing was carried out in a number of steps with slurries of progressively smaller grain size from 3-0.05 $\mu \mathrm{m}$. The slurries were made by suspending alumina powders in mineral oil. Each mechanical polishing step causes some surface damage as well, which extends into the crystal bulk to a depth of approximately three times the size of the powder being used [20]. Therefore, progressively 
smaller powders are used to reduce the surface damage. Some of the detector crystals were then etched with a $2 \% \mathrm{w} / \mathrm{v}$ solution of $\mathrm{KI}$ in water. The surfaces of the treated detectors were then examined under an optical microscope and compared to the untreated surfaces.
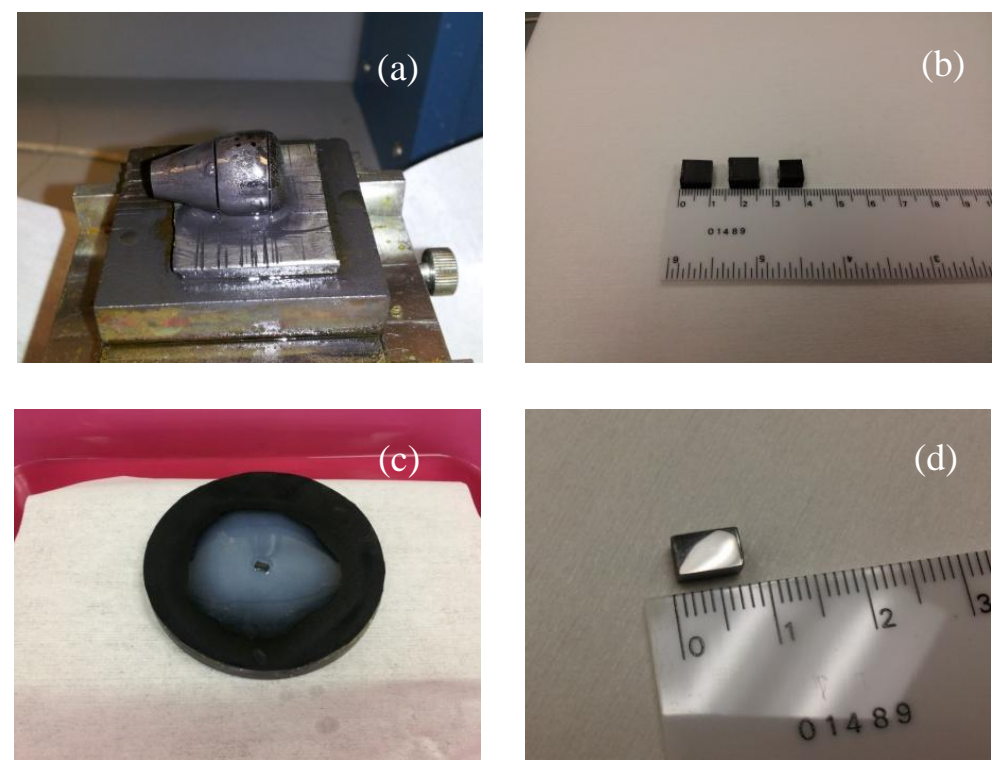

Figure 2. Steps in the detector fabrication process, showing the (a) wire saw cutting, (b) detector crystals prior to polishing, (c) mechanical polishing, and (d) detector crystal after polishing.

Two opposite cleaved surfaces of the detector crystals were sputter coated with Au metal using a Kurt J. Lesker® CMS-18 multi target sputter system. The thickness of the sputtered electrode was around $100 \mathrm{~nm}$. The sputtering was conducted in a vacuum chamber with a pressure of $10^{-8}$ Torr, at a deposition rate of approximately $0.5 \mathrm{~nm} / \mathrm{s}$ and at room temperature. Some detectors had planar electrodes for both anode and cathode side, while a few were fabricated such that their anode electrode consisted of a single $1 \mathrm{~mm} \times 1 \mathrm{~mm}$ pixel and the cathode was deposited as a planar electrode. The pixel electrode geometry was chosen to exploit the small pixel effect to achieve single polarity charge sensing [21].

\section{Detector Characterization}

The main purpose of the surface treatments was to reduce the detector leakage current. Leakage current measurement was therefore performed to determine the variation of leakage current flowing through the device to understand the behavior of the detector under a constant bias for prolonged times. The detectors are connected in series with a Keithley 6517B electrometer, which supplies voltage and measures the current through the detector as a function of time.

Radiation responses of the $\mathrm{BiI}_{3}$ detectors were investigated using Amptek A250 charge sensitive pre-amplifiers and standard NIM electronics. Spectrum acquisition was carried out using an ORTEC ${ }^{\circledR}$ EASY-MCA and Maestro 32 spectrum acquisition software. The detector, 
preamplifiers, and the radiation source were all enclosed in a grounded aluminum box (Figure 3). This shields the detectors and the sensitive preamplifiers from light and other electromagnetic interference. The radiation response measurements were carried out at room temperature.

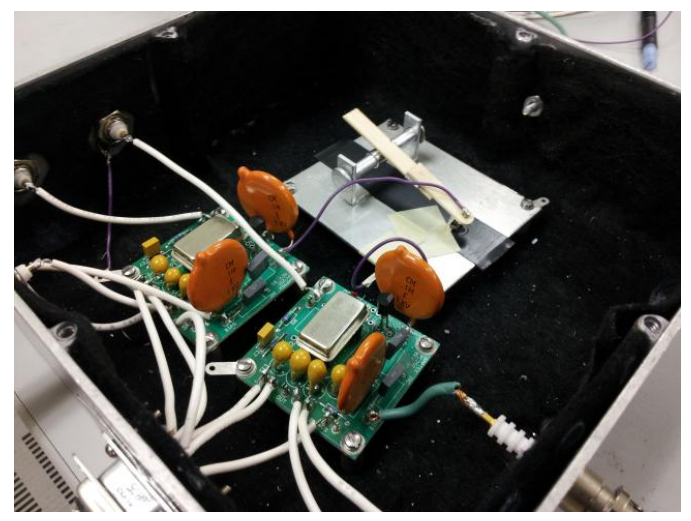

Figure 3. Aluminum test box with a $\mathrm{BiI}_{3}$ detector connected to the preamplifiers.

\section{Results and Discussion}

Optical microscopy images were obtained for the treated surfaces and can be seen in Figure 4. The roughness and defects resulting from the wire saw cutting can be seen on the untreated surface of a detector in Figure 4(a). The surfaces of the untreated detector crystal were rough and dark gray colored. The treated surfaces on the other hand were visibly smoother and more uniform than the untreated surface.
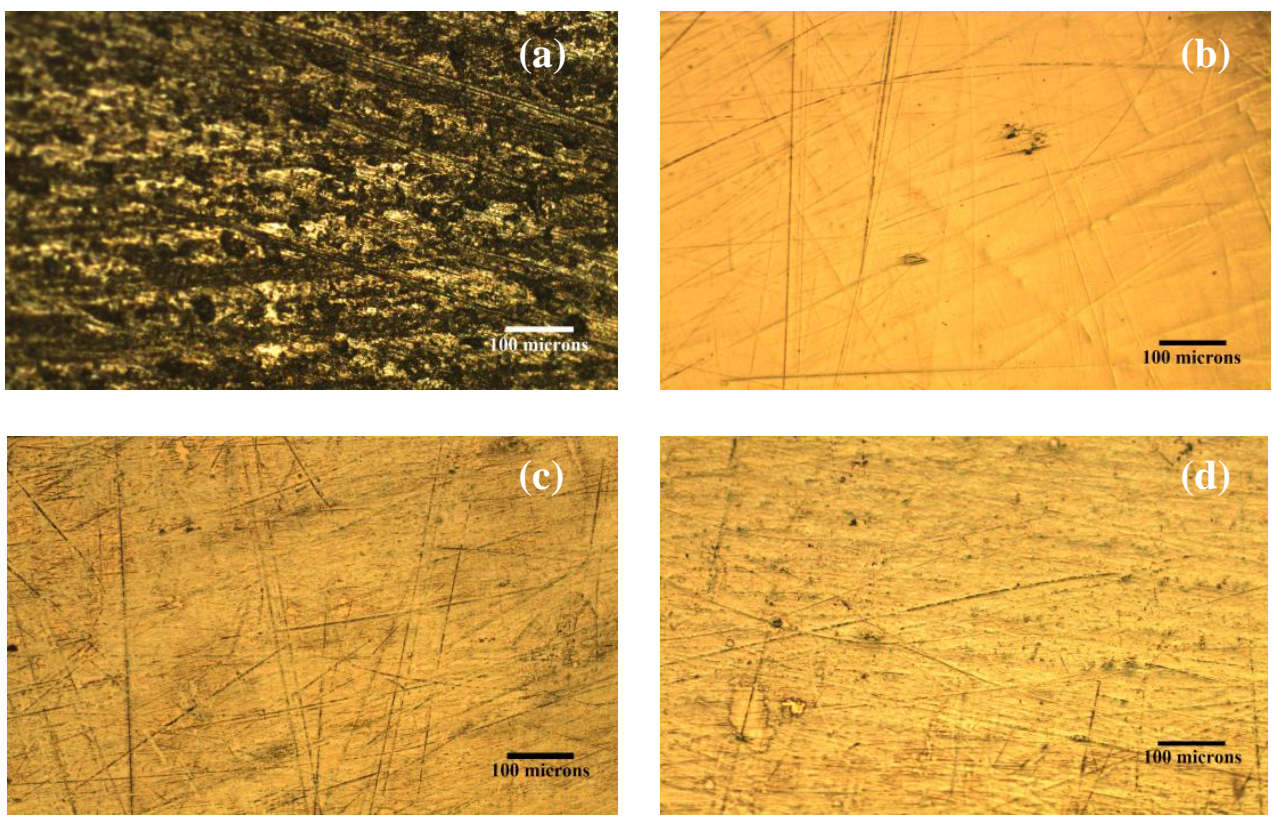

Figure 4. Optical microscopy images of $\mathrm{BiI}_{3}$ detector crystals subjected to (a) no polishing or etching (b) only polishing (c) polishing and etching with $2 \% \mathrm{w} / \mathrm{v}$ KI solution for $30 \mathrm{sec}$. (d) polishing and etching with $2 \% \mathrm{w} / \mathrm{v}$ KI solution for $1 \mathrm{~min}$. 
Since the crystals are relatively soft, the polishing was carried out manually at slow speed to reduce the risk of deep scratches and chips or other structural damage that could be caused due to mechanical stress. There were some scratches and hollows that were kept visible on the treated surfaces. These scratches could be caused due to excessive and uneven pressure being applied during polishing; however they could also be caused during cutting or they could be the result of alumina being too hard an abrasive for $\mathrm{BiI}_{3}$. Even with the scratches, the surface treatments were successful in removing the major surface defects and the overall performance of the detectors was improved.

The effect of the surface treatment on the device leakage current was observed by measuring the leakage current under a constant bias voltage over an extended period of time. A comparison of the leakage current density vs. time for four different detectors can be seen in Figure 5. The detectors were once again biased at a constant electric field of $100 \mathrm{~V} / \mathrm{cm}$. Of the four detectors, three were subjected to different surface treatments with the exception of a reference untreated detector. Surface treatment may improve the surface resistivity, which in turn may cause a decrease in the surface leakage current and thereby reduce the overall leakage current of the detectors. It can be observed that the detectors with surface treatment had significantly lower leakage current density than the untreated detectors.

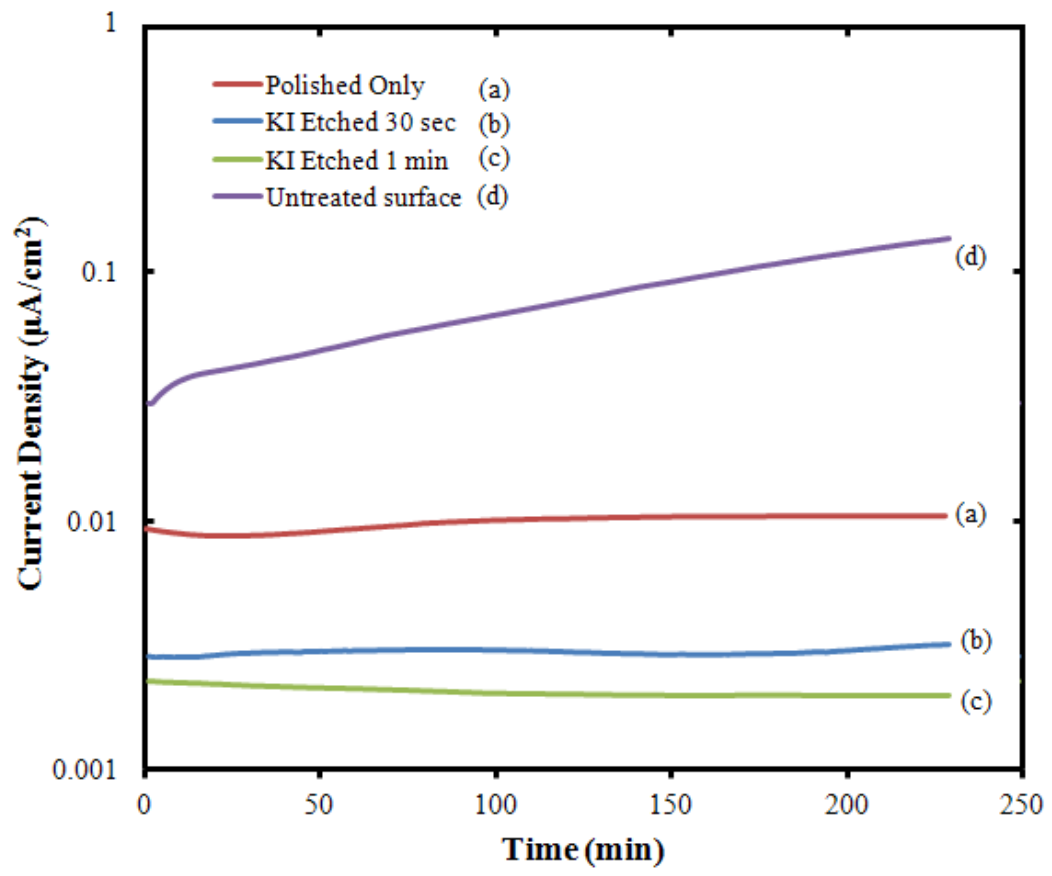

Figure 5. Time dependent leakage current behavior of the different detectors. The detectors were biased at $100 \mathrm{~V} / \mathrm{cm}$.

It can be seen that the leakage current plots shown in Figure 5 do not reproduce the same shape corresponding to the plot shown in Figure $1 \mathrm{~b}$ for the $0.5 \mathrm{at} \% \mathrm{Sb}_{\text {doped }} \mathrm{BiI}_{3}$ crystal. The exact cause for the different shape is not yet known. Unlike the samples used for Figure 5, the crystal samples used for the leakage current measurements shown in Figure $1 \mathrm{~b}$ were prepared by cutting 
the crystal with a razor blade, which may have induced internal defects in addition to forming very rough surfaces. Therefore, since the leakage current may be influenced by both internal defects and surface morphology, the leakage current profiles are not exactly reproduced by the different samples.

The influence of etching was studied by measuring the leakage current before and after subjecting the detectors to etching. The leakage current in the polished detectors prior to etching was comparable to that observed for other detectors which had been polished but not etched. The lateral sides of the detectors were then etched with the $2 \% \mathrm{w} / \mathrm{v} \mathrm{KI}$ solution. Care was taken to ensure that the electrode surfaces were not affected and only the lateral sides were etched. Some detectors were subjected to etching for 30 seconds, while others were etched for 1 minute. For both cases it was observed that the detectors had lower leakage current after etching. Figure 6 shows a comparison of the leakage current prior to etching and after etching for two different detectors.

Typically it was observed that the detectors with treated lateral surfaces had lower leakage current. Several detectors with and without surface treatment were prepared for this study. However, not all detectors treated or otherwise produced a measurable response to radiation. Low leakage current alone does not guarantee a good radiation response. The reasons for this randomness in the $\mathrm{BiI}_{3}$ detector radiation response needs further investigation. Currently it is hypothesized that the radiation response may be affected by the concentration of defects within the detector crystals.

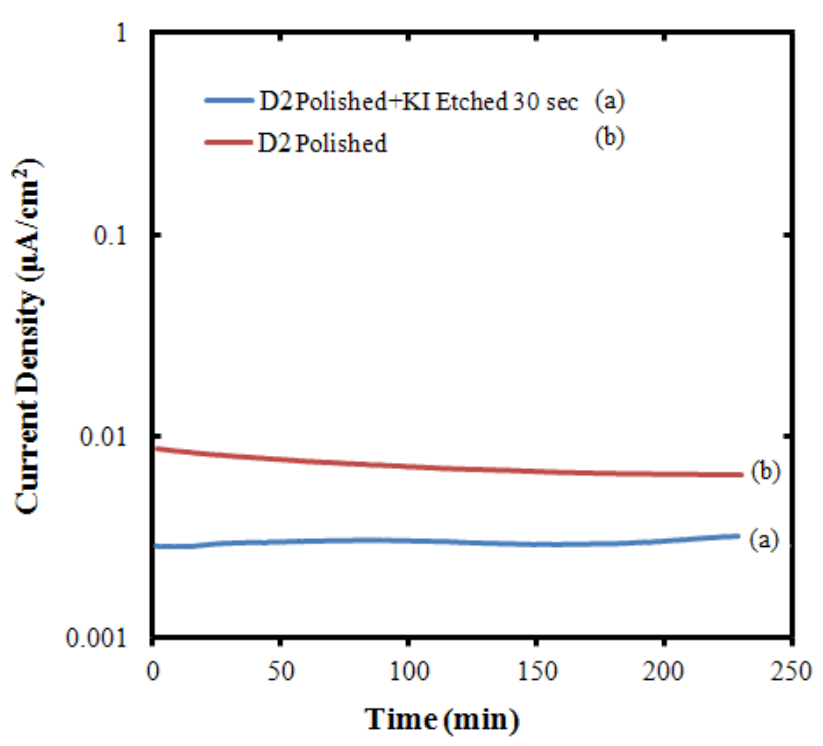

(a)

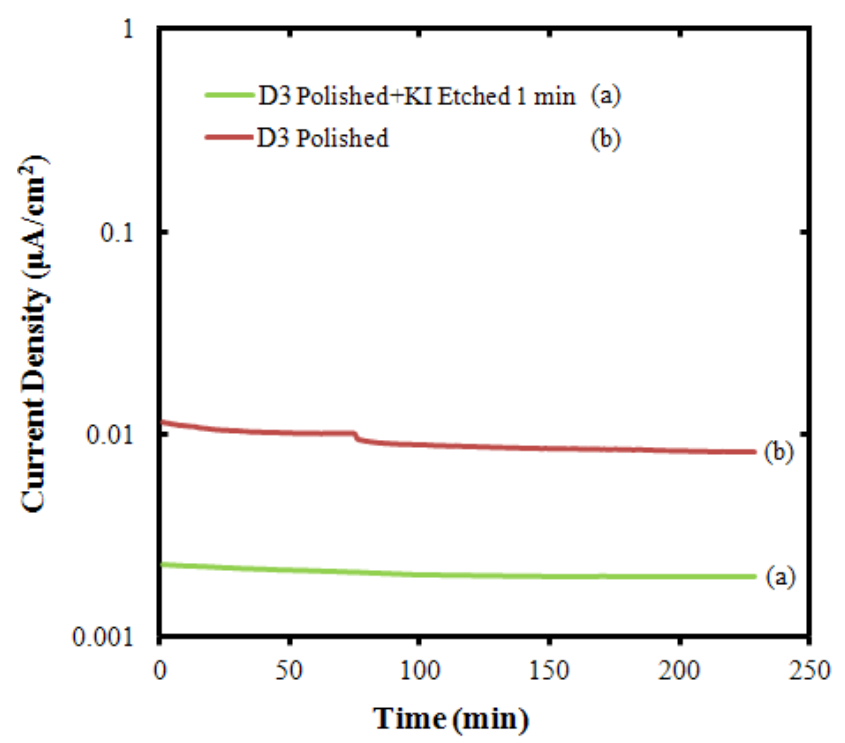

(b)

Figure 6. Leakage current vs. time measurement of detectors before and after etching (a) detector subjected to etching for $30 \mathrm{sec}$ (D2), (b) detector subjected to etching for $1 \mathrm{~min}$ (D3).

The following results focus on only those detectors which produced a radiation response. The performance of each of the detector was further evaluated by measuring the radiation response. The detector cathode was irradiated with gamma-rays from a $1 \mu \mathrm{Ci}^{241} \mathrm{Am}$ radiation source. A 
negative biasing voltage was applied to the cathode and pulse height spectra for the detectors were recorded by collecting the output signal off of the detector anode. Table 1 shows the various parameters for the detectors that are the focus of this manuscript.

Table 1. List of detectors

\begin{tabular}{|c|c|c|c|c|c|c|}
\hline \multirow[t]{2}{*}{ Detector } & \multirow{2}{*}{$\begin{array}{c}\text { Crystal } \\
\text { Dimensions } \\
(1 \times \mathrm{w} \times \mathrm{h} \mathrm{cm})\end{array}$} & \multirow[t]{2}{*}{$\begin{array}{l}\text { Surface } \\
\text { Treatment }\end{array}$} & \multicolumn{2}{|c|}{$\begin{array}{l}\text { Electrode Type/ } \\
\text { Electrode surface } \\
\text { dimensions } \\
(1 \times \mathrm{w} \mathrm{cm})\end{array}$} & \multirow[t]{2}{*}{$\begin{array}{c}\text { Operating } \\
\text { Bias (V) }\end{array}$} & \multirow{2}{*}{$\begin{array}{l}\text { Leakage } \\
\text { current at } \\
100 \mathrm{~V} / \mathrm{cm}^{2} \\
\left(\mu \mathrm{A} / \mathrm{cm}^{2}\right)\end{array}$} \\
\hline & & & Anode & Cathode & & \\
\hline D1 & $0.5 \times 0.3 \times 0.4$ & Polishing & $\begin{array}{c}\text { Planar } \\
(0.5 \times 0.3)\end{array}$ & $\begin{array}{l}\text { Planar } \\
(0.5 \times 0.3)\end{array}$ & 203 & $\sim 0.01$ \\
\hline D2 & $0.3 \times 0.5 \times 0.5$ & $\begin{array}{l}\text { Polishing and } \\
\text { etching for } 30 \mathrm{~s}\end{array}$ & $\begin{array}{c}\text { Planar } \\
(0.3 \times 0.5)\end{array}$ & $\begin{array}{l}\text { Planar } \\
(0.3 \times 0.5)\end{array}$ & 118 & $\sim 0.002$ \\
\hline D3 & $0.5 \times 0.5 \times 0.2$ & $\begin{array}{l}\text { Polishing and } \\
\text { etching for } 1 \\
\text { min }\end{array}$ & $\begin{array}{c}\text { Pixel } \\
(0.1 \times 0.1)\end{array}$ & $\begin{array}{l}\text { Planar } \\
(0.5 \times 0.5)\end{array}$ & 185 & $\sim 0.002$ \\
\hline D4 & $0.6 \times 0.2 \times 0.4$ & $\begin{array}{c}\text { No surface } \\
\text { treatment }\end{array}$ & $\begin{array}{c}\text { Planar } \\
(0.6 \times 0.2)\end{array}$ & $\begin{array}{c}\text { Planar } \\
(0.6 \times 0.2)\end{array}$ & 213 & $\sim 0.1$ \\
\hline
\end{tabular}

Detector D1 was subjected to mechanical polishing and it had planar electrodes for both the cathode as well as the anode. The thickness of the detector between the electrodes was $0.4 \mathrm{~cm}$. The spectra recorded with detector D1 can be seen in Figure 7. The purpose of this measurement was to compare the background spectrum to a gamma-ray spectrum measured by the same detector. This was to gauge the reliability of the spectra recoded by the detector.

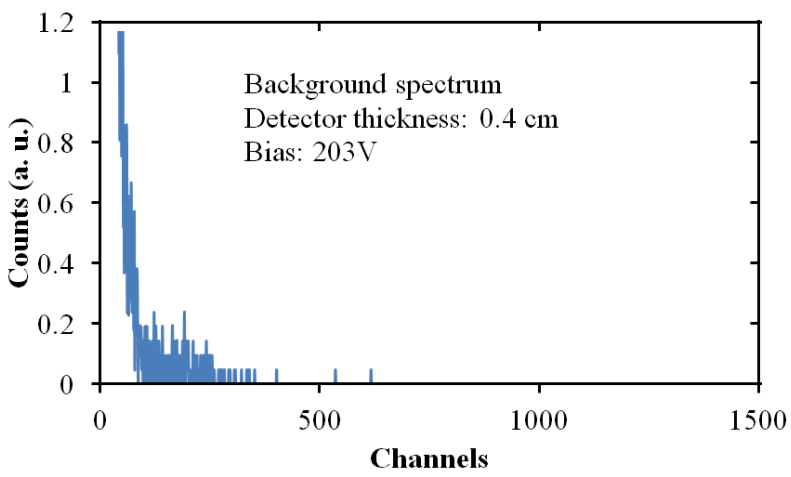

(a)

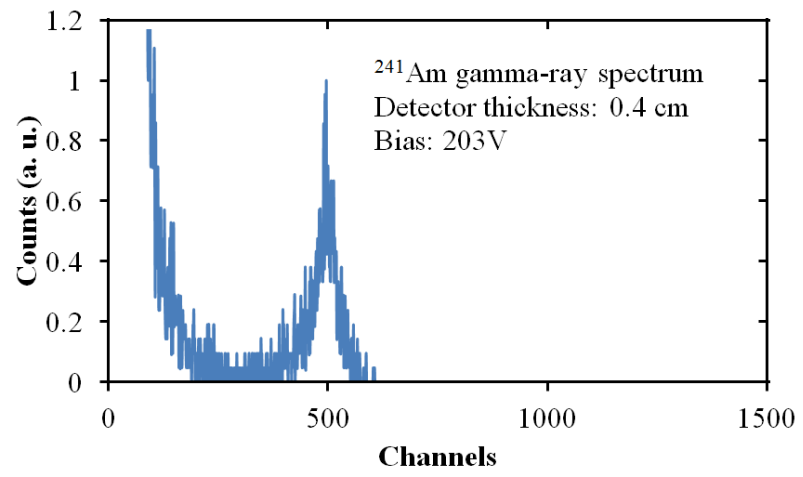

(b)

Figure 7. Spectra obtained using detector D1, (a) Background spectrum with no radiation source, (b) ${ }^{241} \mathrm{Am}$ gamma-ray spectrum. The spectra were recorded at room temperature. 
Both the background spectrum and the gamma-ray spectrum were recorded at the same detector bias of $203 \mathrm{~V}$. The background spectrum was acquired before the gamma-ray spectrum in order to minimize the influence of polarization if any, on the spectrum. A clear difference was observed between the two spectra. The gamma-ray spectrum exhibited a peak around channel 500 , and as expected the background spectrum did not have any peaks.

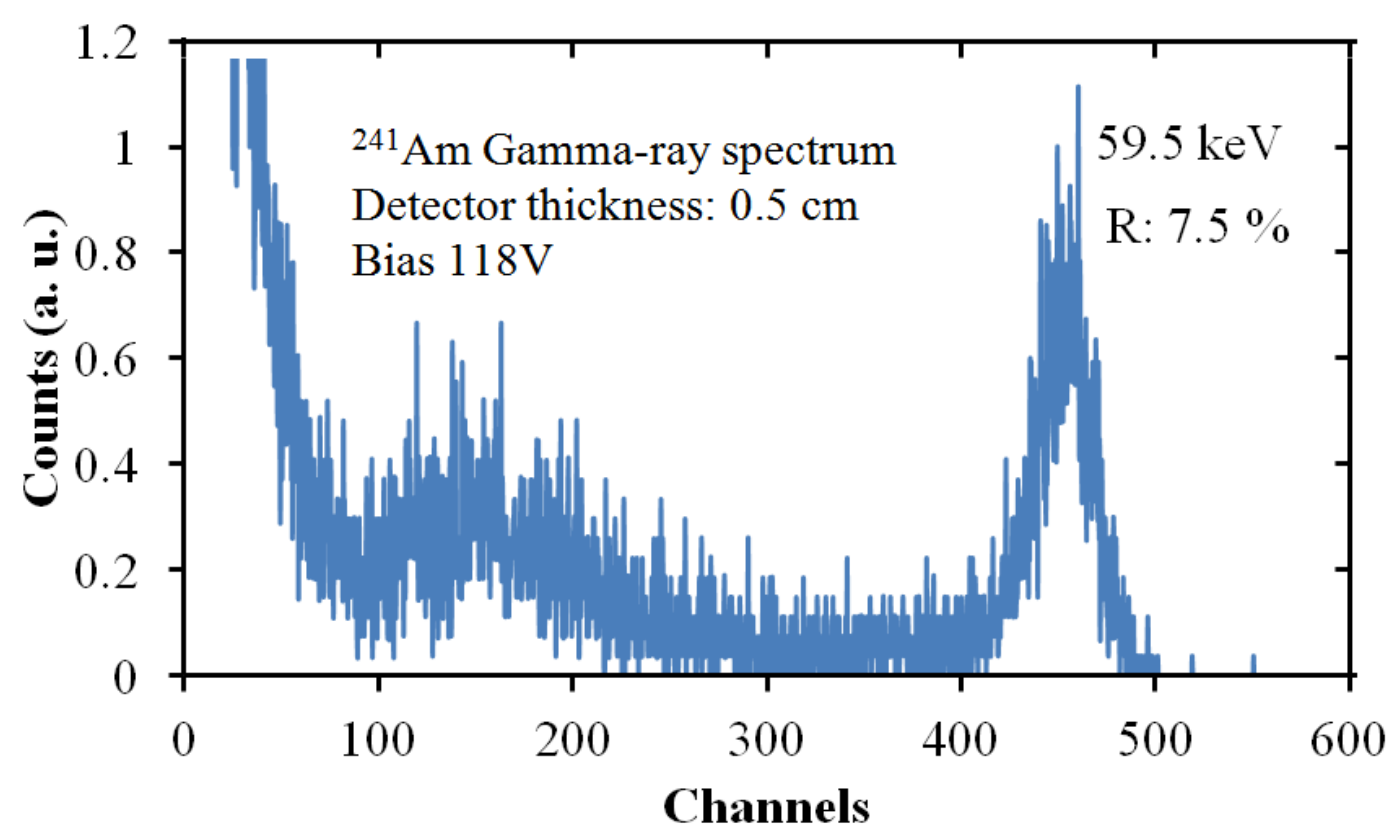

Figure 8. ${ }^{241}$ Am gamma-ray spectrum obtained from detector D2. The spectrum was recorded at room temperature.

The spectra recorded using detector D2 can be seen in Figure 8. This detector had been subjected to mechanical polishing as well as chemical etching with $2 \% \mathrm{w} / \mathrm{v}$ solution of KI for 30 seconds. The leakage current in the detector before and after etching can be seen in Figure 6(a). The detector had planar electrodes for both anode as well as cathode and its thickness between the electrodes was approximately $0.5 \mathrm{~cm}$. It was operated at room temperature at a bias voltage of $118 \mathrm{~V}$. The detector exhibited a full energy peak with a resolution of $7.5 \%$. The counts below the $59.5 \mathrm{keV}$ peak may be due to a combination of the low energy x-rays, noise, and variations in the electric field within the detector. Some semiconductor detectors exhibit a transient behavior where, initial spectra show some noise fluctuations and over time the spectra recorded by the detectors become uniform as the detector stabilizes. [22] - [23]. 


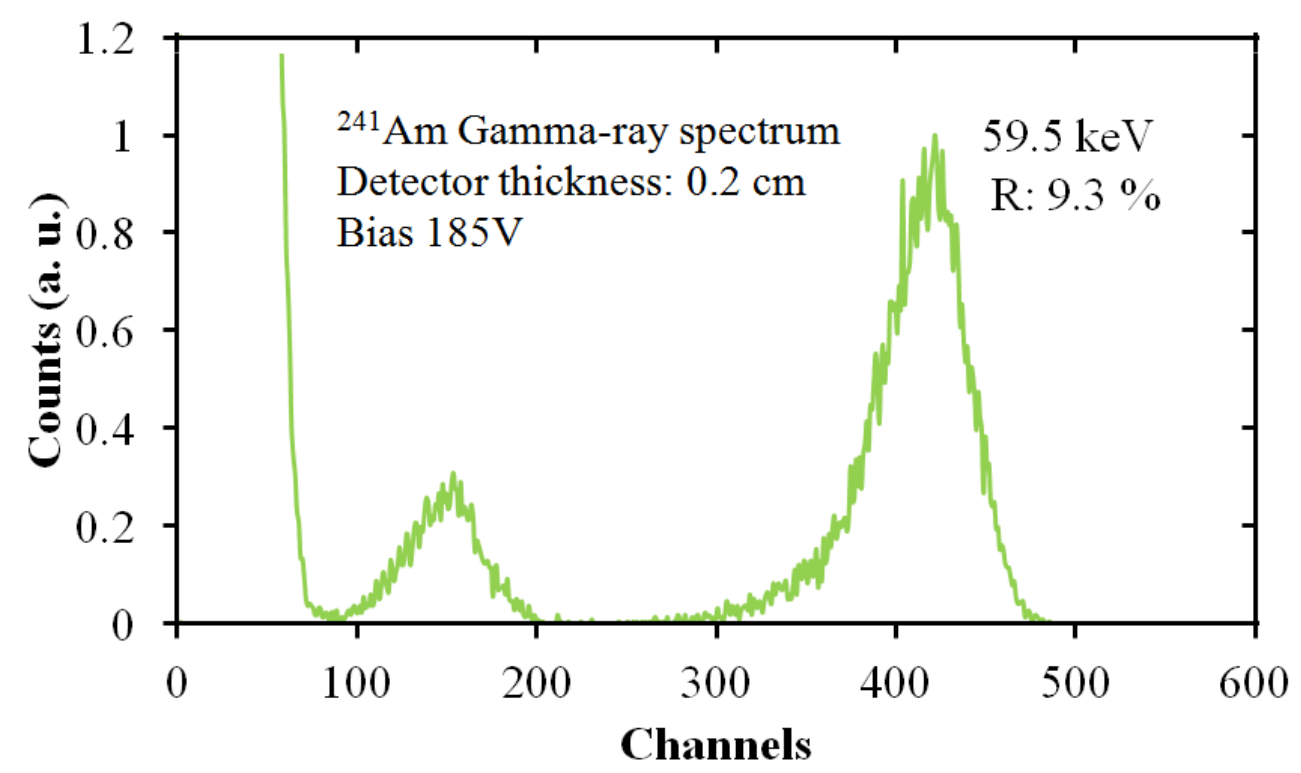

Figure 9. ${ }^{241}$ Am gamma-ray spectrum obtained from detector D3. The spectrum was recorded at room temperature.

The spectrum recorded using detector D3 can be seen in Figure 9. The detector was polished and etched with $2 \% \mathrm{w} / \mathrm{v}$ KI solution for 1 minute. The leakage current in the detector before and after etching can be seen in Figure 6(b). The anode consisted of a $1 \mathrm{~mm} \times 1 \mathrm{~mm}$ pixel electrode and the thickness of the detector between the electrodes was $0.2 \mathrm{~cm}$; and it was operated at room temperature and the applied bias voltage was $185 \mathrm{~V}$. The detector exhibited a full energy peak with a resolution of $9.3 \%$. Once again the counts below the $59.5 \mathrm{keV}$ peak, between channels $100-200$ may be due to the low energy x-rays. The spectra in both Figure 8 and Figure 9 show this low energy x-ray peak (or combination of peaks), however the spectrum for detector D1 shown in Figure 7 does not exhibit this low energy structure. This may be because the noise variation in D1 may have been too high and as a result the signal generated by the low energy $\mathrm{x}$ rays may have been lost in the noise.

The above spectra are especially important since $\mathrm{BiI}_{3}$ response to gamma-rays has not been reported previously. Since all the spectra were recorded by irradiating the detector cathodes with low energy gamma rays, it can be safely assumed that most of the gamma-ray interactions occur close to the cathode. As such, the movement of the electrons has a greater contribution to the detector output signal as compared to holes. This is true even for the detectors with a planar anode (D1, D2).

The mobility-lifetime product $(\mu \tau)$ for detector D4 was estimated by recording the channel number of full energy peak at two different bias voltages. The detector had not been subjected to any surface treatment. The leakage current in the detector can be seen in Figure 5, plot (d). The detector cathode was irradiated by an alpha particle source and the spectra were recorded at two bias voltages (Figure 10). Alpha particles have very low penetrating power; hence it can be assumed that most of the interactions occur near the cathode surface. An alpha spectrum was 
then recorded at a detector bias of $174 \mathrm{~V}$; the detector bias was then increased to $220 \mathrm{~V}$ so that there was a significant shift in the full energy peak.

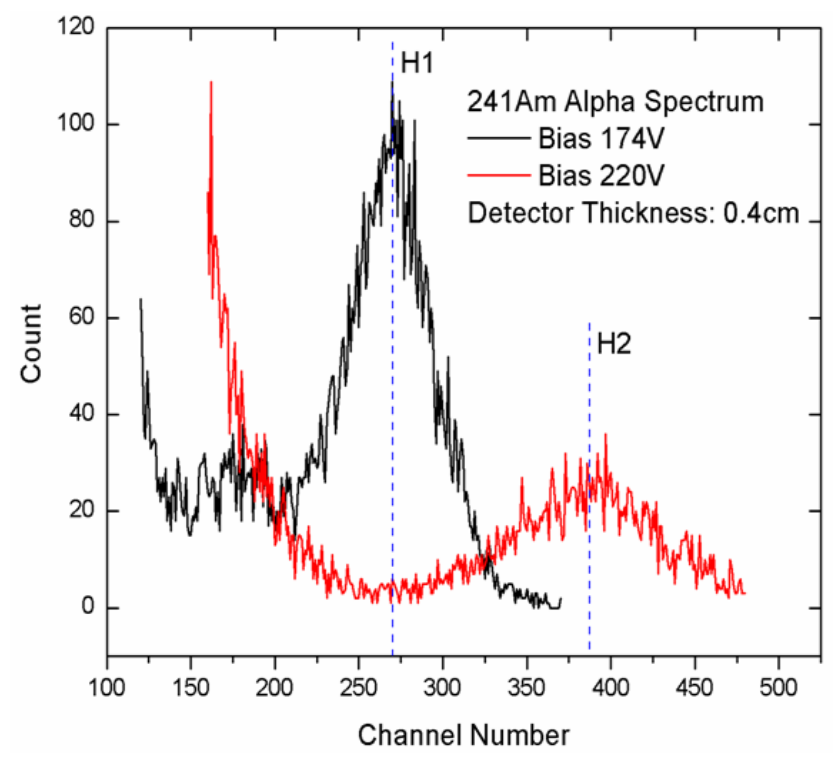

Figure 10. Alpha spectra obtained using a $0.4 \mathrm{~cm}$ thick $\mathrm{Sb}$ doped $\mathrm{BiI}_{3}$ detector (detector D4) at two different bias voltages. The shift in alpha peaks was used to estimate the $(\mu \tau)$ product for electrons.

This shift in the full energy peak was then used to estimate the $\mu \tau$ product using the following equation

$$
(\mu \tau)_{e}=\frac{D^{2}}{\ln \left(\frac{H_{1}}{H_{2}}\right)}\left(\frac{1}{V_{2}}-\frac{1}{V_{1}}\right)
$$

$D$ is the detector thickness $(0.4 \mathrm{~cm}), V_{l}$ and $V_{2}$ are two different applied bias voltages, and $H_{1}$ and $H_{2}$ are the full energy peak centroid recorded with the detector under the two bias voltages respectively.

The cathode of the detector was irradiated with $5.48 \mathrm{MeV}$ alpha particles from a ${ }^{241} \mathrm{Am}$ source of $10 \mu \mathrm{Ci}$ activity. Varying the bias voltage causes the amount of charge trapping to vary, which causes the spectra to shift. The shift in spectrum was then used to determine the $\mu \tau$ product. The $\mu \tau$ product was estimated to be approximately $5.2 \times 10^{-4} \mathrm{~cm}^{2} / \mathrm{V}$. Using this value along with the previously estimated value of the electron mobility (approximately $1000 \mathrm{~cm}^{2} / \mathrm{Vs}$ [9]), the lifetime of the electrons was estimated to be approximately $0.54 \mu \mathrm{s}$. The value of $\mu \tau$ product can vary from detector to detector since it depends on the concentration of internal defects and traps. Taking this into consideration the estimated electron $\mu \tau$ product for the $\mathrm{Sb}$ doped $\mathrm{BiI}_{3}$ is comparable to that reported for $\mathrm{HgI}_{2}\left(\sim 10^{-4}-10^{-3} \mathrm{~cm}^{2} / \mathrm{V}\right)$ and $\mathrm{TlBr}\left(\sim 10^{-4} \mathrm{~cm}^{2} / \mathrm{V}\right)$, but is lower than that reported for CZT $\left(\sim 10^{-3} \mathrm{~cm}^{2} / \mathrm{V}\right)$ [24], [25], and [26]. The low $\mu \tau$ product of the electrons could be attributed to the presence of internal defects and trapping sites within the 
crystal. Due to the charge trapping the hole $\mu \tau$ could not be measured because the output signal due to holes was very low. This would suggest the holes are more susceptible to trapping than electrons.

The alpha particles do not penetrate far into the detector bulk and deposit most of their energy near the surface. Therefore, the detector cathode irradiation with alpha particles causes the generation of charge carriers in the region very close to the cathode surface. Thus, the low value of the $\mu_{e} \tau_{e}$ may be explained as an artifact due to the distortion of the electric field at the surface. Since the holes do not travel as far as the electrons, their movement will be affected by the surface conditions more than the electrons, which also explains why the hole $\mu \tau$ product could not be estimated by the alpha particle irradiation. Further characterization and verification of the electron and hole $\mu \tau$ products using different techniques will be performed in future works.

After a time period of 24 hours; the detector D4 was once again subjected to radiation response measurement. A bias of $213 \mathrm{~V}$ was applied under which the detector operated for around 6 hours. During this time ${ }^{241} \mathrm{Am}$ alpha particle spectra were acquired at different time intervals. It was observed that the counting efficiency of the detector decreased significantly with time. The detector operational conditions such as bias voltage, ambient temperature, source strength, etc. were constant during the length of the experiment. This suggests that the decline in the detector performance was caused due to polarization effects. It can be seen from Figure 11 that both the peak positions as well as the count rate decrease with time. This is consistent with the effects of polarization where the buildup of trapped charges over time creates an opposing electric field. This prevents the charge carriers from moving towards the electrodes and causes a loss in output signal.

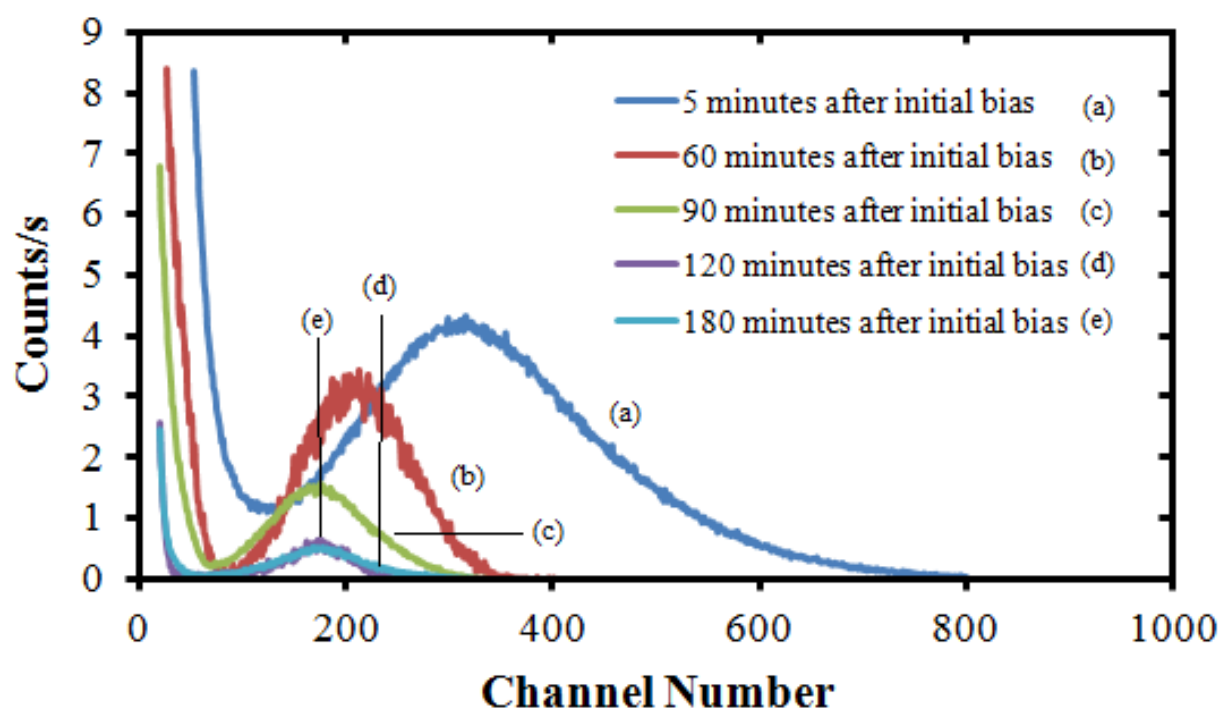

Figure 11. ${ }^{241} \mathrm{Am}$ alpha particle spectra recorded at various time intervals at room temperature with the detector D4. The shift in the spectra is an indication of detector polarization. 
The initial decline in the counting efficiency was sharp and the channel number of alpha peak decreased by half in the first 90 minutes of operation, from approximately 360 to 170 . As the time progresses the decrease in the detector performance slowed down between $90-120$ minutes from initial bias. The counting efficiency was constant between $120-180$ minutes after initial bias; however later it deteriorated slowly until the detector stopped producing output pulses around 6 hours after initial bias. In our previous work, it was reported that the undoped $\mathrm{BiI}_{3}$ detector stopped working after approximately 90 minutes under bias [13]. The $\mathrm{Sb}$ doped $\mathrm{BiI}_{3}$ detector on the other hand operated for a longer time. The detector polarization along with the low mobility-lifetime product of electrons indicates that charge trapping has a significant effect on the detector performance. Further investigation is required to study the nature of these charge trapping sites in $\mathrm{BiI}_{3}$ : $\mathrm{Sb}$ and to possibly develop ways to mitigate their effects.

\section{Summary}

Maiden spectral response of $\mathrm{BiI}_{3}$ detectors to gamma-rays is here reported. It was observed that the electrical characteristics, especially the resistivity of $\mathrm{BiI}_{3}$ can be enhanced by doping the crystals with 0.5 at\% Antimony ( $\mathrm{Sb}$ ). A detector fabrication protocol involving surface treatment was implemented. The importance of mechanical and chemical surface treatments was demonstrated from the results obtained in this work.

All the detectors exhibited similar morphology before surface treatment and an improvement in the surface morphology and detector performance was observed after subjecting the detectors to various surface treatments.

The detectors prepared by the successful implementation of the Sb doping and the fabrication protocol, produced the first response to gamma-rays by $\mathrm{BiI}_{3}$ detectors. ${ }^{241} \mathrm{Am}$ gamma-ray spectra were recorded at room temperature with an energy resolution of $7.5 \%$ at $59.5 \mathrm{keV}$. Detectors with different electrode geometries were prepared and tested. Detectors with pixelated anode mitigate the dependence of detector output on hole transport. The room temperature response to gamma-rays provides incentive to work towards furthering the development of $\mathrm{BiI}_{3}$ detectors for a number of applications such as remote field operation.

The mobility-lifetime product for electrons was estimated to be approximately $5.2 \times 10^{-4} \mathrm{~cm}^{2} / \mathrm{V}$, while the hole trapping prevented the measurement of the hole mobility-lifetime product. The effect of charge trapping could possibly be reduced by further improving the crystal growth process to produce crystals with low internal defects and trapping sites.

\section{Acknowledgements}

This project was supported by the Department of Energy National Nuclear Security Administration under contract DE-FG52-09NA29358.

VI. References 
[1] M. Matsumoto, K. Hitomi, T. Shoji and Y. Hiratate, Bismuth tri-iodide crystal for nuclear radiation detectors, IEEE Trans. Nucl. Sci. 49 (2002) 2517.

[2] W. Qiu, B. B. Hinojosa, X. Haixuan, M. A. Motyka, S. R. Phillpot, J. E. Baciak, S. TrolierMcKinstry, and J. C. Nino, Band gap and structure of single crystal $\mathrm{BiI}_{3}$ : Resolving discrepancies in literature, J. Appl. Phys. 114 (2013) 033110.

[3] M. S. Goorsky, H. Yoon, M. Schieber, R. B. James, D. S. McGregor, M. Natarajan,X-ray diffuse scattering for evaluation of wide bandgap semiconductor nuclear radiation detectors, Nucl. Instr. Meth. A 380 (1996) 6.

[4] L. Fornaro, I. Aguiar, A. Noguera, M. Perez, and M. Rodriguez, Improvements on Bismuth Tri-Iodide platelets for room temperature x-ray detection, IEEE NSS Conf. Rec. R04-3 (2006) 3616.

[5] Y. Cui, M. Groza, A. Burger, and R. B. james, Effects of surface processing on the performance of $\mathrm{Cd}_{1-\mathrm{x}} \mathrm{Zn}_{\mathrm{x}}$ Te radiation detectors, IEEE Trans. Nucl. Sci. 51 (2004) 1172.

[6] L. Fornaro, I. Aguiar, N. Sasen, M. Perez, and A. Noguera, Improving the detection performance of heavy metal halides films by surface treatment, IEEE NSS Conf. Rec. (2010) 3793.

[7] I. B. Oliveira, F. E. Costa, P. K. Kiyohara, and M. M. Hamada, Influence of crystalline surface quality on TlBr radiation detector performance, IEEE Trans. Nucl. Sci. 52 (2005) 2058.

[8] Q. Zheng, F. Dierre, J. Crocco, V. Carcelen, H. Bensalah, J. L. Plaza, and E. Dieguez, Influence of surface preparation on CdZnTe nuclear radiation detectors, App. Surf. Sci. 257 (2011) 8742.

[9] H. Han, M. Hong, S. S. Gokhale, S. B. Sinnott, K. A. Jordan, J. E. Baciak, and J. C. Nino, Defect engineering of BiI3 single crystals: enhanced electrical and radiation performance for room temperature gamma-ray detection, J. Phys. Chem. C 118 (2014) 3244.

[10] L. Fornaro, E. Saucedo, L. Mussio, A. Gancharov, and A. Cuna, Bismuth triiodide polycrystalline films for digital X-ray radiography applications, IEEE Trans. Nucl. Sci. 51 (2004) 96.

[11] A. Cuna, N. Noguera, E. Saucedo, and L. Fornaro, Growth of bismuth tri-iodide platelets by the physical vapor deposition method, Cryst. Res. Technol. 39 (2004) 912.

[12] I. Aguiar, S. Kroger, and L. Fornaro, Bismuth tri-iodide polycrystalline films for X-ray direct and digital imagers, Nucl. Instr. Meth. A 610 (2009) 332.

[13] A. T. Lintereur, W. Qiu, J. C. Nino, and J. E. Baciak, Characterization of bismuth tri-iodide single crystals for wide band-gap semiconductor radiation detectors, Nucl. Instr. Meth. A 652 (2011) 166.

[14] Y. N. Dmitriev, P. R. Bennett, L. J. Cirignano, M. Klugerman, and K. S. Shah, Bismuth Iodide crystals as a detector material: Some optical and electrical properties, SPIE Proc. 3768 (1999) 521

[15] D. Nason and L. Keller, The growth and crystallography of bismuth tri-iodide crystals grown by vapor transport, J. Cryst. Growth. 156 (1995) 221.

[16] S. S. Gokhale, H. Han, J. E. Baciak, J. C. Nino, K. A. Jordan, Growth, fabrication, and testing of bismuth tri-iodide semiconductor radiation detectors, Radiat. Meas. 74 (2015) 47.

[17] J. P. Ponpon, R. Stuck, M. Amann, Current instability in mercuric iodide devices, Solid-State Electron. 44 (2000) 29. 
[18] K. Hitomi, Y. Kikuchi, T. Shoji, K. Ishii, Polarization phenomenon in TlBr detectors, IEEE. Trans. Nucl. Sci. 56 (2009) 1859.

[19] A. Owens, M. Bavdaz, G. Brammertz, V. Gostilo, H. Graafsma, A. Kozorezov, M. Krumrey, I. Lisjutin, A. Peacock, A. Puig, H. Sipila, and S. Zatoloka, The X-ray response of TlBr, Nucl. Instr.Meth. A 497 (2003) 370.

[20] A. Kargar, A. M. Jones, W. J. McNeil, M. J. Harrison, and D. S. McGregor, CdZnTe Frisch collar detectors for gamma-ray spectroscopy, Nucl. Instr. Meth. A 558 (2006) 497.

[21] H. H. Barett, J. D. Eskin, and H. B. Barber, Charge transport in arrays of semiconductor gamma-ray detectors, Phys. Rev. Lett. 75 (1995) 156.

[22] A. Owens, M. Bavdaz, G. Brammertz, M. Krumrey, D. Martin, A. Peacock, L. Troger, The hard X-ray response of $\mathrm{HgI}_{2}$, Nucl. Instr. Meth. A 479 (2002) 535.

[23] C. L. Thrall, W. R. Kaye, Z. He, H. Kim, L. Cirignano, K. Shah, Transient behavior in TlBr gamma-ray detectors and its analysis using 3D position sensing, IEEE Trans. Nucl. Sci. 60 (2013) 1162.

[24] N. Auricchio, L. Marchini, E. Caroli, A. Zappettini, and L. Abbene, Charge transport properties in CdZnTe detectors grown by the bridgman technique, J. Appl. Phys. 110 (2011) 124502-1.

[25] D. Alexiev, N. Dytlewski, M. I. Reinhard, and L. Mo, Characterization of single-crystal mercuric iodide, Nucl. Instr. Meth. A 517 (2004) 226.

[26] K. Hitomi, M. Matsumoto, O. Muroi, T. Shoji, and Y. Hiratate, Characterization of thallium bromide crystals for radiation detector applications, J. Cryst. Growth. 225 (2001) 129. 